\title{
(Neo-)Marxistische Politische Ökonomie als Gegenprogramm zur Standardökonomik
}

\author{
Hardy Hanappi D
}

Online publiziert: 5. Februar 2019

(C) List-Gesellschaft e.V. 2019

Zusammenfassung Der Beitrag gibt einen kurzen Überblick der Entwicklung politisch-ökonomischer Theorien in Marx' Tradition und stellt darauf aufbauend die Frage nach deren kontemporärem Stellenwert. Dabei werden insbesondere vier Ausprägungen unterschieden: Matrix Marxismus, Makroökonomischer Marxismus, evolutionstheoretischer Marxismus und synthetischer Marxismus.

Schlüsselwörter Dogmengeschichte $\cdot$ Wirtschaftspolitik $\cdot$ Klassentheorie · Dialektische Methode · Ökonomische Modelle

\section{New Marxist Political Economy as a research program contradicting the neo-classical economic mainstream}

\begin{abstract}
This article provides a brief survey on the development of theories of political economy, which follow the tradition of the contribution of Karl Marx. Building on this perspective of Marxism it then is discussed how relevant it is for contemporary problems of political economy. It is argued that its impact can be structured according to four strands of Marxism: Matrix Marxism, Macroeconomic Marxism, Evolutionary Marxism, and Synthetic Marxism.
\end{abstract}

Keywords History of economic thought · Economic policy · Theory of classes · Dialectic method · Economic models

\footnotetext{
H. Hanappi $(\bowtie)$

Institut für Stochastik und Wirtschaftsmathematik, 105-3, TU Wien, Wiedner

Hauptstrasse 6-8, 1040 Wien, Österreich

E-Mail: hanappi@tuwien.ac.at; hanappi@gmail.com
} 
Ökonomische Denkschulen sind keine Religionen. Marxistische politische Ökonomie ist daher auch nicht dadurch betreibbar, dass man in den Schriften des Karl Marx danach sucht was er in Wahrheit gemeint hat um damit die tatsächliche Wahrheit aus dem Geiste des Gründervaters geliefert zu bekommen. Wie bei der gemeinhin als neoklassische Theorie bezeichneten Standardökonomik, die ja ebenfalls ihren Ikonen huldigt (z.B. Walras, Samuelson, Sargent), gibt es bei einer ökonomischen Denkschule stets einen Reihe zunächst nur schwach verbundener Kernargumentationen, deren Hauptmerkmal darin besteht, dass sie einander wesentlich weniger widersprechen als es gegenüber einem konkurrierenden Paradigma ${ }^{1}$ der Fall ist. Bei den Ansätzen in Marxscher Tradition geht es also darum einige der Argumentationsstränge zu sammeln, die alle ein dafür typisches Element enthalten, das andererseits in konkurrierenden Denkschulen unterbelichtet bleibt.

Um dieses aufzufinden ist es nötig ein wenig in die Entwicklung des Denkens von Karl Marx anhand der Biographien des Mannes einzutauchen ${ }^{2}$. Zunächst war Marx Rheinländer, Jus-Student und Junghegelianer. Ein großer Teil seines Denkens beschäftigte sich mit der Überwindung religiöser Ideologie, Napoleons Eroberung Europas hatte mit politischer Gewalt den Geist der französischen Aufklärung in den Köpfen vieler Gelehrter hinterlassen. Selbst der preußische Feudalstaat war geneigt Elemente des aufgeklärten Absolutismus zuzulassen. Die philosophische Speerspitze der Folgen der Aufklärung in deutschen Landen waren Kant und Hegel. Insbesondere Letzterer war prägend für das Denken des jungen Marx, an seiner Denkweise schulte sich Marx. Hegel hatte die fortschreitende Negation von bereits Negiertem in endlos langen Sätzen zelebriert und in diesem Prozess die Entstehung neuer Ideen, wenn schon nicht sichtbar, so doch fühlbar gemacht. Die Universalität dieser Vorgangsweise war in Frankreich bereits von Descartes zum Motto der Aufklärung erklärt worden: „Da du nicht leugnen kannst, daß du zweifelst, und es im Gegenteil gewiß ist, daß du zweifelst, und zwar so gewiß, daß du daran nicht zweifeln kannst, so ist auch wahr, daß du, der du zweifelst, bist, und dies ist auch in solcher Weise wahr, daß du nicht mehr daran zweifeln kannst.“ (Descartes 1631) ${ }^{3}$. Wie Descartes hatte auch Hegel seine Methode aber trotz strenger Anwendung in eine Lobpreisung Gottes münden lassen - in das Gegenteil ihrer impliziten Zielsetzung. Im damaligen politischen Umfeld wäre jede andere Position streng sanktioniert worden.

Marx nützte Hegels Denkweise, landläufig als Dialektik bekannt, und „stülpte sie um“ (Althusser 2005). In gleichsam evolutionstheoretischer Weise ${ }^{4}$ versuchte er das Wesen des denkenden Individuums aus seinen materiellen Lebensumständen abzuleiten anstatt den Prozess als ein sich Materialisieren abstrakter - letztlich göttlicher

\footnotetext{
${ }^{1}$ Das Wort „Paradigma“, also „gegen das Gesagte“ bringt diese gegensätzliche Bewegung - Kohäsion nach innen Adhäsion nach außen - ganz gut zum Ausdruck.

2 Aus der Vielzahl der Biographien seien hier nur zwei neuere genannt: Jones (2016) und Neffe (2017)).

3 Descartes wird mit dieser Methode auch zum Begründer des methodologischen Individualismus: „Was aber bin ich demnach? Ein denkendes Wesen! Was heißt das? Nun, - ein Wesen, das zweifelt, einsieht, bejaht, verneint, will, nicht will und das sich auch etwas bildlich vorstellt und empfindet." (Descartes, 2. Meditation Punkt 8). Dieser war in einer Zeit religiöser Dominanz ein erster progressiver Schritt zum Verständnis gesellschaftlicher Dynamik.

${ }^{4}$ Interessant ist diesbezüglich auch der freundliche Briefwechsel zwischen Marx und Darwin, siehe (Gould 2011).
} 
- Prinzipien aufzufassen. Der Zweifel, der in den internen Modellen der Menschen auftretende Widerspruch, entsteht demnach im tatsächlich geführten Leben. Das Grundelement von Marx, entlang dem sich auch alle hier folgenden Argumentationslinien bewegen, bleibt aber die Beschreibung prozessierender Widersprüche und die damit einhergehende Entstehung von Neuem.

Die Zeit vor der gescheiterten bürgerlichen Revolution von 1848 war für das methodische Vorgehen von Marx zeitlebens prägend. Dialektik beinhaltet einerseits das strenge Element wiederholender Negation, aber andererseits führt keine dieser Negationen zurück zum Ausgangspunkt, sondern generiert stets auch Neues. Damit beinhaltet sie auch ein Element der Arbitrarität, beinahe schon der Beliebigkeit, womit sie dem was wir unter Zeit verstehen sehr nahekommt. In den Revolutionswirren um 1848 ist Marx als Politiker zu vielen kurzfristig nötigen Einschätzungen und Entscheidungen gezwungen. Hier stellt sich die Frage des revolutionären Subjekts: Wer ist der Agent, der die gesellschaftlichen Widersprüche schubweise auflöst und neue Konstellationen durchsetzt? Marx bricht mit dem methodologischen Individualismus seiner philosophischen Lehrer und führt den Begriff der ökonomischen Klasse ein. Die Geschichte ist eine Folge von prozessierenden Klassenwidersprüchen, wobei neue Klassen entstehen und alte in den Hintergrund geraten. Sein Favorit als langfristig progressivster Agent ist das Proletariat. Die Koalitionsproblematik zwischen Proletariat, bürgerlicher Klasse und Feudalklasse steht im Zentrum der Strategiebildung. Er entdeckt die Tücken der Konflikte die sich zwischen kurz- und langfristigen Dynamiken, zwischen koalitionsfördernder Toleranz und publikumswirksamer Unnachgiebigkeit auftun. Er ist gezwungen sorgsame Analyse durch Intuition zu ersetzen, hat manchmal recht, irrt aber auch oft. Unter Stress verkommt Dialektik leicht zu seichter Polemik - auch das eine historische Warnung. Letztlich scheitert die Revolution. Marx muss flüchten und wieder folgt er in London einer alten Hegelschen Maxime: Im Konzept der Aufhebung nützt Hegel den Doppelsinn der deutschen Sprache um im selben Prozess sowohl die Eliminierung als auch die Bewahrung des bestehenden Zustands auszudrücken - selbstredend ein Widerspruch. Und zwar folgendermaßen. Der in die British Library verbannte Marx zieht nach der gescheiterten Revolution den Schluss, dass seine Theorie nicht entwickelt genug war, auch den, dass er vielleicht nicht genug an profundem ökonomischen Wissen aufgehoben - im Sinne von bewahrt - hat ${ }^{5}$. Marx studiert die klassischen britischen politischen Ökonomen im Detail und unterscheidet deren Erkenntnisse scharf von den Schriften von „Vulgärökonomen“. Die daraus zu ziehende Lehre ist, dass auch dialektische Theoriebildung der schubweisen Dynamik zwischen Erarbeitung und Bewahrung wertvollen Wissens und intuitiv-innovativer Revolutionierung nicht entkommen kann. Das Ergebnis der Einarbeitung der britischen Klassiker in seine zuvor eher intuitive Identifizierung des Proletariats als revolutionäres Subjekt ist der erste Band von „Das Kapital“. Marx bleibt seiner dialektischen Vorgangsweise treu und beginnt mit dem am meisten entfremdeten und doch zugleich vertrautestem Element des Alltagslebens - der Ware. Das ist einerseits ein durchaus didaktisches Vorgehen, andererseits ist aber auch schon der erste grundlegende Widerspruch für

\footnotetext{
5 In seinen öffentlichen Auftritten, ja nicht einmal Engels gegenüber hätte Marx das zugegeben. Es ist nur spekulativ an seinen Handlungen ablesbar.
} 
spätere Marxinterpretationen angelegt. Im Begriff der Ware begegnet sich - ganz im methodischen Sinne Hegels - Erscheinungsform und Wesen eines offensichtlichen Grundelements des Kapitalismus. Was erscheint ist einerseits ein immer unübersichtlicher „Warenbrei“ an materiellen Gütern und Dienstleistungen, den ein sehr langer Preisvektor begleitet. Als Wesen versteckt sich hinter diesen beiden Phänomenen der Mechanismus der kapitalistischen Produktionsweise, präziser gesagt, wie aus dem Produktionszusammenhang die beobachteten Warenmengen und Preise entstehen. Eine wichtige Aufgabe der Wissenschaft der politischen Ökonomie ist es eben dieses Wesen aufzudecken. Marx Grundidee ist hierbei, dass jeder einzelnen Ware neben dem empirisch am Markt zu beobachtenden Preis auch ein sozialer Wert zugeordnet werden kann. Dieser gesamtgesellschaftlich verankerte Wert (und hier folgt Marx den klassischen britischen politischen Ökonomen) wird letztlich von den in die Herstellung der Ware eingehenden Arbeitszeiten bestimmt - ,Am Ende ist alle Ökonomie, Ökonomie der Zeit“". Zu beachten sind dabei jedenfalls die folgenden Punkte:

1. Durchschnittsbildungen für eine bestimmte Warenart sind unumgänglich, da ja vielfältige Produktionsprozesse parallel laufen und auch unterschiedliche Bedürfnisse der Konsumenten in die letztlich auf den Märkten auftretenden Preise dieser Ware eingehen. Das soll zunächst als Aggregationsproblem bezeichnet werden.

2. Arbeitszeiten sind insofern heterogen als dem einzelnen Individuum unterschiedliche Wertigkeit der aufgewendeten physikalischen Zeit zugerechnet werden muss, abhängig davon wie gut es in der Lage ist die gestellte Arbeitsaufgabe zu erfüllen. Diese Problematik wird traditionell Reduktionsproblem (auf einfache Arbeit) genannt.

3. Der Fortschritt der menschlichen Produktivkraft erfolgt durch fortschreitende Teilung - und damit einhergehender Notwendigkeit immer neuer Integration und Kombination $^{6}$ - der heterogenen Arbeitszeitbeiträge. Hierin ist bereits angelegt, dass eine Teilung der Gesellschaft in Klassen durch das Auseinanderfallen der sozialen Macht, also der Entscheidungsgewalt darüber wie die Produktionsprozesse zerteilt und wieder zusammengesetzt werden, in warenproduzierenden Gesellschaften unumgänglich ist. Letztlich läuft dieses Problem auf den berühmten $W i$ derspruch zwischen Produktivkräften und Produktionsverhältnissen hinaus: Jede herrschende Klasse ist bemüht ihre Entscheidungsmacht selbst dann noch zu erhalten, wenn die Möglichkeiten einer Veränderung der Klassenstruktur dem Fortschritt zuträglicher wären. Das weist natürlich über die Thematik „Ware“ hinaus auf ein Studium langfristiger historischer Klassendynamik.

4. Besonders für die von der Entscheidungsgewalt ausgeschlossenen Klassen tritt mit zunehmender Arbeitsteilung auch zunehmendes Unverständnis der fremdbestimmten gesamtgesellschaftlichen Entwicklung - Entfremdung - ein. Aber auch die herrschende Klasse selbst sieht sich in den ihr durch die Produktionsverhältnisse aufgezwungenen Verhaltensweisen gefangen. Nicht zufällig nennt Marx sein

\footnotetext{
6 Die neue Kombination von Produktionsprozessen ermöglicht nicht nur eine Einsparung von Zeit zur Produktion von bestehenden Warentypen, sondern auch die Einführung neuer Waren. Es ist gerade diese gesteigerte systematische Innovationfähigkeit die die historische Mission des Kapitalismus ausmacht.
} 
Buch „Das Kapital“ und nicht „Die Kapitalisten“, es ist der Systemzusammenhang, der die Menschen in ihr Verhalten zwingt und sie so zu seinem Anhängsel macht ${ }^{7}$.

5. Mit zunehmender Entfremdung wird aber auch das Problem der ursprünglich von Marx angenommenen engen Bindung zwischen ökonomischer Stellung (Arbeiter, Kapitalist) und dem Bewusstsein davon, dem Klassenbewusstsein, immer problematischer. Die einzelnen Klassen beginnen entlang ihrer vom ökonomischen Status zunehmend entkoppelten Bewusstseinslagen zu zerfallen. Am deutlichsten wird dieser Gedankengang bei Marx allerdings vor seinem Hauptwerk, im Text „Zur Judenfrage“ ausgeführt. Hier wird nahezu prophetisch - man denke an den Faschismus des 20. Jahrhunderts - die Projektion kapitalistischer Verhaltensweise auf eine eher arbiträr ausgewählte Bevölkerungsgruppe, die Juden, dargestellt. Die Verbindung zwischen Sein und Bewusstsein, die eine Klasse ausmacht, verlagert sich nicht zuletzt auf Grund moderner Informationstechnologien immer stärker in Richtung einer Dominanz des Bewusstseins. Auch diese Klassenproblematik weist natürlich weit über den Begriff der Ware hinaus ${ }^{8}$.

Marx selbst hat diese mehrschichtigen, komplizierten Problemkreise nicht einmal ansatzweise gelöst, er hat aber höchst wortreich und eindringlich viele Details zu Tage gebracht und Argumentationslinien vorgezeichnet.

Wodurch er sich dabei vom heutigen Mainstream unterscheidet kann besser verstanden werden, wenn man betrachtet wie die Gründerväter des heutigen Mainstreams, die Marginalisten, ab 1874 - noch zu Lebzeiten Marx - mit diesen Problemen umgingen.

Der zentrale politische Ausgangspunkt des von Jevons, Menger und Walras vertretenen damals neuen Ansatzpunktes besteht darin die in (5) skizzierten Implikation auszublenden. Die von den klassischen britischen Ökonomen vertretene politische Ökonomie wird reduziert auf ein wissenschaftliches Gebiet, das sich Ökonomie nennt. Der politische Rahmen, die Machtstruktur, in dem sich Letztere bewegt wird an eine andere Wissenschaft (Politikwissenschaft) delegiert und somit ein davon unabhängiger Bereich der Wissenschaft postuliert: Das, was später Mikroökonomie genannt wird und sich am Atomismus der theoretischen Physik und Newtons Formalismen orientiert. Mit den ewig gültigen Eigenschaften der ,ökonomischen Atome" - des homo economicus und der repräsentativen Firma - kann die historisch offene Klassendynamik durch statische Glaubenssätze ersetzt werden. Ein Bild, das die Gemütslage der durch die gescheiterte Revolution von 1848 in das politische System des aufgeklärten Absolutismus integrierte Bourgeoisie durchaus traf. Einerseits wird unterstrichen, dass das alte Feudalsystem unnütz ist. Es kommt in dieser Theorie der Ökonomie nicht vor. Doch genau dadurch entfernt sich der

\footnotetext{
7 Im Vorwort zur zweiten Auflage spricht Marx von den „Charaktermasken“, die der kapitalistische Prozess seinen Betreibern aufzwingt.

8 Es ist interessant zu sehen, dass der vom Scheitern der 1848 Revolution enttäuschte Marx in London neues Wissen zu akkumulieren sucht indem er mehr klassische Ökonomie studiert. Dem Bestreben Althussers erst diesen Marx als Wissenschaftler anzuerkennen wäre aber festzuhalten, dass es auch in seiner Londoner Zeit das Festhalten an seiner mit Widersprüchen arbeitenden Dialektik - ein Erbe Hegels - ist, die die innovativsten theoretischen Ideen erzeugt hat.
} 
Marginalismus von jedweder praktischen Realität, denn im wirklichen Leben schaltet und waltet selbstverständlich stets ein politischer Staatsapparat, ist untrennbar mit den Aktionen der ökonomischen Atome verbunden. Von daher, vom Angriff auf die politische Dimension der klassischen politischen Ökonomie, rührt also die bis heute offene Wunde der wirtschaftspolitischen Impotenz der Standardökonomie. Als besänftigtes Anhängsel an den feudalen Herrscher ist es dem 1848 erschrockenen Bürger auch nicht mehr so wichtig die politisch Mächtigen durch gewagte neue Gesellschaftsentwürfe herauszufordern. Erst nachdem die Feudalsysteme im ersten Weltkrieg zusammenbrechen und die große Depression der 30er Jahre die selbstverstärkenden Krisenprozesse in sogenannten ,freien Marktwirtschaften“ drastisch vor Augen führt, wird der Ruf nach einer Wiedereinführung staatlicher politischer Praxis und sie leitender Theorie laut. Das ist denn auch die Stunde der anderen Hälfte der Standardökonomie, der Makroökonomie. Keynes, der Architekt dieser Halbwelt, bietet zunächst eine Scheinlösung für Problem (1), das Aggregationsproblem, an: Anstatt wie bisher theoretisch vorzugehen nähme man einfach an, es gäbe ein staatliches, statistisches Amt, das alle quantitativen Summanden erhebt und summiert. Es ist dabei unwichtig wie bei jedem Individuum und jeder Firma der jeweilige ökonomische Fingerabdruck zustande gekommen ist (das kann im mikroökonomischen Wolkenkuckucksheim mathematisch diskutiert werden); wichtig ist einzig, dass den Aggregatgrößen ein (zumeist monetärer) Wert zugeordnet werden kann. Einfache Buchhaltungsidentitäten auf nationaler Ebene ermöglichen es dann den Staat als neuen, weitgehend klassen-neutralen Akteur in den Kreislauf der aggregierten Stromgrößen wiederum einzuführen. Problemkreis (4) wird von Keynes eliminiert indem von vornherein festgestellt wird, dass es sich bei der Makroökonomie nur um eine Theorie der kurzen Frist handelt. Ihre Momentaufnahmen ${ }^{9}$ lassen die mehrere Jahrhunderte dauernde dynamische Entwicklung des Kapitalismus völlig unerklärt. Erst die Nachfolger von Keynes füllen diese Lücke indem sie die Statik zwischen den absoluten aggregierten Werten durch eine statische Struktur der Wachstumsraten dieser Aggregate, einen Gleichgewichtspfad, ersetzen. Das gelieferte Ergebnis ist dann die Menge von gleichgewichts-kompatiblen Wachstumspfaden der Aggregate statt wie davor Gleichgewichtswerte der Aggregate selbst ${ }^{10}$.

Nach der Veröffentlichung des ersten Bandes von „Das Kapital“ ist Marx intensiv damit beschäftigt die dort eher intuitiv und konzentriert versammelten Ideen vertieft und mit breiterem historischen Hintergrund $\mathrm{zu}$ fundieren, zum Beispiel in den ,Theorien über den Mehrwert“, aber auch der posthum herausgegeben 3. Band des „Kapitals“ belegt das. Dabei zeigt sich, dass die Materie viel komplizierter ist als es die im Schwange der 48-er Revolution nötigen polemischen Überspitzungen vermuten ließen. Es ist daher wenig überraschend, dass diese späten Auseinandersetzungen und ihre Unstimmigkeiten der Angelpunkt für die frühe Kritik an Marx - etwa von Böhm-Bawerk (Böhm-Bawerk 1896) - sind. Bei der Wiederentdeckung

\footnotetext{
9 Im Vergleich zur mehrere hundert Jahre dauernden Produktionsweise Kapitalismus ist die für 3 bis 5 Jahre dauernde Kurzfristigkeit der Makroökonomie tatsächlich ein „Moment“.

10 Demgemäß verlagert sich die Diskussion konsistenten Accountings inklusive Staat in Richtung Stabilität von Gleichgewichtspfaden, von eher instabilem (e.g. Harrod 1939) bis zu stabilem Wachstum (e.g. Solow, 1956).
} 
von Marx nach der weltweiten Jugendrevolte von 1968 waren es zunächst genau diese Widersprüche um deren Marx-konforme Korrektur es zu gehen schien.

Im Mittelpunkt standen die ersten beiden Problemkreise - allerdings bereits in dem ihnen von Bortkiewicz (Bortkiewicz 1906) verpassten Gewande: Das Transformationsproblem (wie werden Arbeitswerte zu Preisen?) und das Reduktionsproblem (wie kann komplizierte Arbeitszeit auf einfache Arbeitszeit umgerechnet werden?). Ein guter Teil der als Neomarxismus bezeichneten Ansätze hat hier ihren Ausgangspunkt genommen. Sie basieren in der Regel auf Leontieffs Input-Output Darstellung der Marxschen Reproduktionsschemata ${ }^{11}$. Auf die dabei behandelten Widersprüche wird im Kapitel „Matrix Marxismus“ genauer eingegangen.

Akzeptiert man hingegen die kurzgeschlossene Argumentationsweise von Keynes bezüglich Aggregation, so stellt sich der von ihm dargestellte Widerspruch zwischen aggregiertem Konsum, aggregiertem Sparen und aggregierter Investition als eine Frage des Verhältnisses zwischen sozialpsychologischen Konstanten, den Parametern der makroökonomischen Verhaltensfunktionen dar. Keynes Vorgehen kann damit insofern als ein Schritt in Richtung Marx verstanden werden als bei ihm durchaus krisenhafte Ungleichgewichte entstehen können, die dann nur durch maßgeschneiderte Intervention eines neuen exogenen Agenten - des Staates - korrigiert werden können. Insbesondere durch den Übergang zur Wachstumstheorie und die Einbeziehung von Erwartungsprozessen haben die Epigonen von Keynes einige Flanken der keynesianischen Theorie aufgezeigt, die mehr als nur die Rettung des Kapitalismus durch Staatsintervention darstellbar machen. Der neomarxistischen Interpretationen von Widersprüchen in Keynes makroökonomischem Kontext wird im Kapitel „Makroökonomischer Marxismus“" nachgegangen.

In den beiden soeben genannten Annäherungen an Marx' Gedankengänge kommt allerdings der in (3) genannte wichtige Aspekt des Widerspruchs zwischen Produktivkräften und Produktionsverhältnissen nicht vor. Schumpeter, Zeitgenosse von Keynes, hatte Marx nicht nur genauer als Keynes studiert, sondern hatte auch genau diesen Widerspruch als Ausgangspunkt seiner ökonomischen Theorie gewählt: Den im Gleichgewicht (oder im gleichgewichtigen Wachstum) befindlichen repräsentativen Firmen und Haushalten ${ }^{12}$ wird ein Typ von Akteur eingepflanzt, der Unternehmer, der durch kreative Zerstörung das gesamte System überhaupt erst antreibt. Was durch den Erfolg - trotz zeitweiligem Scheitern - dieses neuen Charakterdarstellers in der Gesellschaft geschaffen wird ist Produktivkraft. Und da sie zugleich Althergebrachtes zerstört stellen sich ihr die herrschenden Produktionsverhältnisse entgegen. Der neue Wind, der hier schubweise in die erstarrenden Verhältnisse geblasen wird wurde wirtschaftspolitisch allerdings eher selten als eine moderne

\footnotetext{
11 Wassily Leontieff, politisch gesehen ein Sozialdemokrat, studierte bis 1925 in der Sowjetunion und war daher mit Marx Theorie bestens vertraut. Seine systematische Erfassung der Verflechtung einer Wirtschaft in I-O Tabellen kann daher als Weiterentwicklung der Marxschen Ideen, die wiederum auf den Tableaus von Quesnay basieren (Quesnay 1758), betrachtet werden (Leontieff 1966).

12 Bei Marx sind die herrschenden Produktionsverhältnisse weiter gefasst und beinhalten auch alle zu Institutionen geronnenen üblichen Relationen zwischen Produktionseinheiten, Haushalten und Staatsakteuren. Selbstverständlich fehlt in der klassischen politischen Ökonomie (und bei Marx) auch die bedingungslose Huldigung des Gleichgewichtsbegriffs; sie ist durch die adäquatere Beschreibung eines Oszillierens um übliche Werte ersetzt.
} 
Parallele zu Marx Metapher von Revolutionen als den Schnellzügen der Geschichte gesehen ${ }^{13}$. Dennoch liegt darin wahrscheinlich eine der wichtigsten Weiterentwicklungen eines Marxschen Gedankenganges; wie im Kapitel „Evolutionstheoretischer Marxismus" skizziert wird.

Schließlich bleiben noch die Widerspruchs-Gruppen rund um Punkt (4) und (5). Schon die Betrachtungen worum es hier inhaltlich geht zeigen, dass eine Beschränkung auf eine Diskussion ökonomischer Alternativen zur Standardökonomie hier sicherlich zu kurz greifen würde. Das im Informationszeitalter inzwischen omnipräsent gewordene Phänomen der Entfremdung verlangt Sozialpsychologie und wirkt nachhaltig auf die ökonomische Basis im Verhältnis zum ideologischem Überbau ein, was wiederum die Analyse von Klassenbildung und Klassendynamik grundlegend beeinflusst. Aus der Vielzahl neomarxistischer Themen in diesem Bereich greift das Kapitel „Synthetischer Marxismus“ einige heraus.

\section{Matrix Marxismus}

Michio Morishima hat sehr schön gezeigt wie man aus einer einfachen Input-Output Tabelle der beobachteten quantitativen Produktionsverflechtung eines Jahres Arbeitswerte berechnen kann (Morishima 1973), siehe auch (Roemer 1981).

Sei A die $n \times n$ Matrix der im letzten Jahr beobachteten Inputs, jedes Element $a_{i j}$ sei also die für das Gut i nötige Menge von Gut j gewesen. Des Weiteren sei L der $1 \times \mathrm{n}$ Vektor der Inputs an direkter Arbeitszeit. Der $1 \times \mathrm{n}$ Vektor $\lambda$ der Arbeitswerte ist dann bestimmt durch:

$$
\lambda=\lambda \mathrm{A}+\mathrm{L} \quad \text { daher gilt also } \quad \lambda=\mathrm{L}(\mathrm{I}-\mathrm{A})^{-1}
$$

Dieser Vektor der zuletzt beobachteten Arbeitswerte ist einzig aus den quantitativen Austauschverhältnissen errechnet und hat überdies die Eigenschaft, dass er als Summe aller vorangegangen Übertragungen von Arbeitswerten (bei Unterstellung einer konstanten Matrix A) interpretiert werden kann ${ }^{14}$, denn

$$
L(I-A)^{-1}=L\left(I+A+A^{2}+A^{3}+A^{4} \ldots\right)
$$

Der Austausch von Waren erfolgt in warenproduzierenden Gesellschaften mittels Geld. Der Preisvektor p der Waren (ein Zeilenvektor) muss also so gestaltet sein, dass Produktivität von A sowie Verteilung mittels p und dem Einkommen aus dem Arbeitszeitvektor L gewährleisten, dass zumindest ein Subsistenzgüterbündel b an Waren gekauft werden kann. Normiert man den Preisvektor p derart, dass der Lohn

\footnotetext{
13 Ein Grund dafür ist sicher auch die zynische, politisch konservative Einstellung Joseph Schumpeters gewesen.

14 Diese Transformation in eine geometrische Reihe hat zu einer etwas unglückseligen Diskussion über die tatsächliche historische Existenz von Systemen einfacher Reproduktion geführt. In der Tat geht es aber bloß um eine abstrakte retrospektive Deutung der zuletzt beobachteten Arbeitswerte, siehe (Pasinetti 1977, pp. 66-69).
} 
Tab. 1 Feudale Verhältnisse

\begin{tabular}{lllllll}
\hline & Luxusgüter & Waffen & Agrarprodukte & Infrastruktur & $\begin{array}{l}\text { Bauern und } \\
\text { Handwerker }\end{array}$ & Adel \\
\hline Luxusgüter & $\mathrm{Q}_{11}$ & $\mathrm{Q}_{12}$ & $\mathrm{Q}_{13}$ & $\mathrm{Q}_{14}$ & $\mathrm{~K}_{\mathrm{B} 1}$ & $\mathrm{~K}_{\mathrm{A} 1}$ \\
Waffen & $\mathrm{Q}_{21}$ & $\mathrm{Q}_{22}$ & $\mathrm{Q}_{23}$ & $\mathrm{Q}_{24}$ & $\mathrm{~K}_{\mathrm{B} 2}$ & $\mathrm{~K}_{\mathrm{A} 2}$ \\
Agrarprodukte & $\mathrm{Q}_{31}$ & $\mathrm{Q}_{32}$ & $\mathrm{Q}_{33}$ & $\mathrm{Q}_{34}$ & $\mathrm{~K}_{\mathrm{B} 3}$ & $\mathrm{~K}_{\mathrm{A} 3}$ \\
Infrastruktur & $\mathrm{Q}_{41}$ & $\mathrm{Q}_{42}$ & $\mathrm{Q}_{43}$ & $\mathrm{Q}_{44}$ & $\mathrm{~K}_{\mathrm{B} 4}$ & $\mathrm{~K}_{\mathrm{A} 4}$ \\
Bauern und & $\mathrm{L}_{\mathrm{B} 1}$ & $\mathrm{~L}_{\mathrm{B} 2}$ & $\mathrm{~L}_{\mathrm{B} 3}$ & $\mathrm{~L}_{\mathrm{B} 4}$ & $\mathrm{~L}_{\mathrm{B} 5}$ & $\mathrm{~L}_{\mathrm{A} 6}$ \\
Handwerker & & & & & & $\mathrm{L}_{\mathrm{A} 5}$ \\
Adel & $\mathrm{L}_{\mathrm{A} 1}$ & $\mathrm{~L}_{\mathrm{A} 2}$ & $\mathrm{~L}_{\mathrm{A} 3}$ & $\mathrm{~L}_{\mathrm{A} 4}$ & & \\
\hline
\end{tabular}

gleich 1 ist, dass Preise also in Arbeitszeiteinheiten ausgedrückt werden, dann muss zur prinzipiellen Reproduktionsfähigkeit der Gesellschaft gelten:

$$
p b \leq 1
$$

All das gilt für alle warenproduzierenden Gesellschaften. Wie aber nun der jeweilige Preisvektor mit dem Vektor der Arbeitswerte zusammenhängt und wie sich insbesondere die die Matrix A - die ja die „Produktivkräfte“ ausdrückt - verändert, all das kann erst auf der Ebene der Produktionsweise spezifiziert werden ${ }^{15}$.

Die Frage, die mit diesem Formalismus in der Folge gestellt werden soll ist nun wie der Preisbildungsmechanismus einer bestimmten Produktionsweise, etwa des Feudalismus oder des Kapitalismus, zum „Erscheinen“ einer Preisstruktur führt, die mit ihrem „Wesen“, der Arbeitswertstruktur, systematisch zusammenhängt. Letztlich wollte Marx, Hegel folgend, die Erscheinung sozialen Wertes als Geld aus der Universalität menschlicher Arbeit entwickeln. Der Zwischenschritt mit dem Marx die Brücke zwischen „Arbeit“ und „Geld“ beginnt (in Hegels Diktion: „,das Besondere“) ist die Ware, daher also zunächst der oben dargestellte Formalismus zu warenproduzierenden Gesellschaften.

Jede Produktionsweise ist durch eine bestimmte Teilung der Menschen in Klassen gekennzeichnet. Dadurch ergibt sich etwa für den Feudalismus sofort, dass die letzte Zeile ebenso wie das Konsumgüterbündel sofort in zumindest zwei Zeilen und zwei Bündelspalten geteilt werden muss ${ }^{16}$. Parallel zu den aus Arbeitsteilung generierten Arbeitswerten tritt damit Macht als zentrales Element in die Argumentation ein. Mit der nun stärker spezifizierten Matrix kann damit sowohl Preisbildung als auch die Ausübung direkter Macht dargestellt werden, siehe Tab. $1^{17}$.

\footnotetext{
15 Ein guter Teil der vielen „Widerlegungen“ eines von Marx nie konsistent vorgelegten Wachstumsmodells (,erweiterte Reproduktion“) beruht auf der Vermischung dieser unterschiedlichen Abstraktionsstufen. Ein ausführlicherer Überblick über die Ansätze des Matrix Marxismus findet sich in (Hanappi 1988).

16 ,Zumindest“ deshalb, weil genau die Entstehung des Bürgertums, einer neuen Klasse zwischen Adeligen und gemeinem Volk, zum entscheidenden Wachstum der Produktivkräfte führt, welches die Produktionsverhältnisse des Feudalismus sprengt.

17 Nur nebenbei sei vermerkt, dass etwa auch das Reduktionsproblem durch Hinzunahme neuer Zeilen und Spalten für besser ausgebildete Qualifikationsstufen von Arbeitern lösbar ist.
} 
Für die direkte Machtausübung hat Marx bereits eine Grundidee geliefert. Sie entwickelt sich in drei Schritten: Zunächst werden die Bauern zur Arbeitsleistung auf den Feldern der Adeligen gezwungen, dann wird ihnen ein Teil der Ernte weggenommen, letztlich müssen sie Geldzahlungen leisten. Alle drei Formen sind als Transfers in der Matrixdarstellung unterscheidbar. Falls der Adel überhaupt Arbeitsleistung in der Agrarproduktion leistet $\left(\mathrm{L}_{\mathrm{A} 3}>0\right)$, etwa durch die Jagd, so wird im Frondienst $\mathrm{L}_{\mathrm{B} 5}$ zum Ersatz von $\mathrm{L}_{\mathrm{A} 3}$ oder als Erhöhung von $\mathrm{L}_{\mathrm{B} 6}$ bei Reduktion von $\mathrm{L}_{\mathrm{A} 6}$ darstellbar. Bei der Rente in Form von Abgabe des Ernteertrags wird ein Teil von $\mathrm{K}_{\mathrm{B} 3}$ nach $\mathrm{K}_{\mathrm{A} 3}$ verschoben. Für die Abgabe in Geldform ist die Einführung von Preisen nötig, also die Bewertung der physischen Quantitäten in Tab. 1. Mit einem Preisvektor entlang der sechs Elemente, $\mathrm{p}_{1}$ bis $\mathrm{p}_{6}$, wäre die Geldrente dann eine Reduktion von $\mathrm{p}_{3} \cdot\left(\mathrm{Q}_{31}+\mathrm{Q}_{32}+\mathrm{Q}_{33}+\mathrm{Q}_{34}\right)$, des Einkommens der Bauern und Handwerker, die zu einer gleich großen Erhöhung des Einkommens des Adels führt, der dieses dann entlang seines Konsums, $\mathrm{K}_{\mathrm{A} 1}, \mathrm{~K}_{\mathrm{A} 2}, \mathrm{~K}_{\mathrm{A} 3}, \mathrm{~K}_{\mathrm{A} 4}$, ausgibt.

Direkte Machtausübung ist denn auch die ursprüngliche Form der Ausbeutung. In feudalen Gesellschaften ist Produktion (1.) überwiegend Agrarproduktion begleitet von der Produktion des dazu benötigten Werkzeugs, (2.) Produktion von Waffen zur Ausübung direkter Gewalt ${ }^{18}$, (3.) Produktion einer territorialen Infrastruktur ${ }^{19}$ sowie (4.) Produktion von Luxuswaren für den Adel. Für diese vier Güter und zwei Klassen sieht die Matrixdarstellung wie in Tab. 1 aus. Deren Elemente können grob eingeschätzt werden: Die Bauern werden kaum Luxusgüter konsumieren, der Adel wird wenig Arbeitszeit in die Landwirtschaft einbringen, Waffen werden für Erhalt und Ausbau der Infrastruktur benötigt, die überhaupt die Domäne ist in der sich die Dominanz der herrschenden Klasse am stärksten ausdrückt, etc. Interessant sind auch die vier untersten rechten Elemente der Tabelle in denen sich die Zeitaufteilung der nicht in der Warenproduktion aufgewendeten Zeit befindet.

Die Etablierung einer Währung durch den Adel erlaubt die Flexibilisierung aller Tauschprozesse, weil sozialer Wert in der institutionalisierten Geldform gespeichert werden kann. Damit ist in der Infrastruktur auch die Erhaltung des Geldwesens durch die herrschende Klasse, den Adel, beinhaltet.

Wie die Preise in dieser Produktionsweise zustande kamen sollte Gegenstand historischer Untersuchungen - und nicht abstrakter ,heroischer“ Annahmen - sein. Was aber jedenfalls zu erwarten ist, ist ein Abweichen der relativen Preise von den relativen Arbeitswerten. Schließlich verschiebt sich durch die Machtausübung der herrschenden Klasse die Verteilung der Arbeitszeit in den Vektoren L und zwar in der Regel unabhängig davon wie Preise für die einzelnen Waren jeweils gebildet werden; Preisbildung als Ausgleichsmechanismus zwischen Angebot und Nachfrage ist ja nur eine von vielen Möglichkeiten.

\footnotetext{
18 Neben direkter physischer Gewalt existiert stets auch informelle Gewalt, insbesondere in Form von Religion.

${ }^{19}$ Es ist die Infrastruktur, durch die die bei Marx als Produktionsverhältnis bezeichneten regulierenden Kräfte der herrschenden Klasse die Produktionsweise stabilisieren. Ein Durchbrechen der Produktionsverhältnisse ist daher stets auch eine Revolution der Infrastruktur.
} 
Die gesamtwirtschaftliche Ausbeutungsrate e in Arbeitswerten ist üblicherweise als Quotient zwischen überschüssiger Arbeitszeit und notwendiger Arbeitszeit definiert:

$$
e(b):=\frac{1-A b}{A b}
$$

Sie stellt den Ausgangspunkt für die tatsächlich stattgefundene Ausbeutung bei der noch Korrekturen wegen Änderung der Bestandgrößen (Schuldenstand und Ersparnis) sowie Umverteilung zwischen den Klassen durch Machtprozesse hinzukommt - dar.

Für die Profitrate muss im Feudalismus der Kapitalbegriff auf seine Ursprungsform, den Grundbesitz, zurückgenommen werden; der herrschenden Feudalklasse kann eine Vorform der Profitrate zugeschrieben werden, die der akquirierten Geldmenge pro Hektar territorialem Besitz entspricht.

Dem genauen, durch historisches Wissen bereicherten Studium des Übergangs vom Feudalismus zum Kapitalismus könnte in der Folge eine den Kapitalismus darstellende Tabelle entworfen werden. Der Adel könnte entfallen, die herrschende Klasse wäre in die im globalen Kontext relevant werdenden Teile - etwa internationales Finanzkapital (wie schon von (Hilferding 1910) antizipiert), reiche nationale Kapitalgruppen (eventuell bestimmter Produkte wie „Energie“, „Waffen“), Verwaltung und militärischer Apparat, etc. - zu zerlegen. Auch die Seite der Arbeiter wäre natürlich genauer zu spezifizieren, insbesondere die Kombinationen territorial-kultureller Prägung mit ökonomischer Position wären zu beachten. Mit diesem Vorgehen fließt dann die Machtstruktur zwischen den Klassen in die tabellarische Darstellung ein. Erst dann kann versucht werden aus der Dynamik des Übergangs vom Feudalismus zum Kapitalismus etwas für den Übergang vom Kapitalismus zur nächsten Produktionsweise zu lernen. Eine Darstellung der momentanen, globalen kapitalistischen I-O Verflechtung inklusive Klassendynamik geht über den Rahmen des hier gebotenen Überblicks hinaus.

Das Grundthema von Marx, der prozessierende Widerspruch - hier zwischen Produktivkräften (in A in Relation zu L) und Produktionsverhältnissen (in der Klassenaufteilung bei Inputs und Konsum sowie explizit im Sektor Infrastruktur, der auch Geldwesen und Institutionen enthält), dieser dynamische Grundtenor kann durch die Input-Output Darstellung zusätzlich erhellt werden. Dabei ist es aber im Gegensatz $\mathrm{zu}$ den meisten gleichgewichtsorientierten Verwendungen des I-O-Ansatzes eben wichtig Entstehung und Verschwinden von Klassen, Sektoren und deren Elementen explizit ernst zu nehmen und empirisch beobachtbaren Wandel einzubauen.

Der Übergang vom Feudalismus zum Kapitalismus hatte mehrere Vorbedingungen, die es erlaubten und erzwangen den Produktivkraftschub durch Welthandel (das Verbinden nationaler I-O Systeme zu einem Imperium) und Militär in ein systematisches Verbessern der Elemente von A (Prozessinnovationen) und Einfügen neuer Zeilen und Spalten (Produktinnovationen) zu transformieren. Das I-O System liefert ein interessantes Grundgerüst über dem diese Charakteristika des Kapitalismus dynamisch eingebaut werden können. 
Neben dieser optimistischen Sicht, für die erst eine Menge Neuland beschritten werden muss, gibt es allerdings auch eine Reihe von Sackgassen in die sich der Matrix Marxismus verlaufen kann. Wie stets, macht es wenig Sinn die wahre Interpretation in Marx Schriften zu suchen und deren Gültigkeit allein aus der Autorität des Autors zu behaupten ${ }^{20}$.

Ebenso hinfällig ist es mit starken Gleichgewichtsannahmen, z. B. mit gleichen sektoralen Profitraten, zu mathematisch ,schönen“ und eindeutigen Lösungen voranzuschreiten zu denen das System dann konvergiert. Eine recht umfangreiche Literatur amerikanischer Provenienz kann über die methodische Beschränktheit allerdings nicht hinwegtäuschen. In einem neueren Artikel versucht Roberto Veneziani sich von der extremen Variante eines „Rational Choice Marxism (RCM)“ zu distanzieren und plädiert für einen offeneren „Analytical Marxism (AM)“ (Veneziani 2010). Bei Betrachtung von Venezianis Definition von AM zeigt sich aber sofort, dass auch dieser noch tief im neoklassischen Denken verhaftet ist. Im Gegensatz zu AM weicht die „Temporal Single-System Interpretation (TSSI)“ von Bortkiewicz Marx Interpretation $\mathrm{ab}$ und betrachtet das Transformationsproblem als inexistent ${ }^{21}$. Es handle sich um ein einziges für den Kapitalismus charakteristisches System (,single system“ und nicht unterschiedliche Systeme für Arbeitswerte und Preise) und dieses sei historisch zu spezifizieren (daher ,temporal“). Die weiter oben präsentierte Darstellung steht daher dem TSSI Ansatz näher, unterscheidet sich von diesem aber dadurch, dass kein Wert darauf gelegt wird zu beweisen, dass Marx Schriften konsistent seien. Es wäre ja aus Sicht dialektischer Logik überhaupt erst festzustellen was unter „Konsistenz“ $\mathrm{zu}$ verstehen ist.

Hier ist festzuhalten, dass die typische Sequenz dialektischer Beschreibung, nämlich Divergenz - Strukturbruch (Entstehung von Neuem) - neue Konsolidierung Divergenz, anzuwenden ist. Als Gegengift für den Irrweg konvergierender Systeme bietet sich der weiter unten beschriebene evolutionstheoretische Marxismus an. Ganz generell ist gerade in der momentanen Situation, dem bevorstehenden Umbruch der globalen politischen Ökonomie, der Rückblick auf historische Umbrüche und dessen Nutzung für die Interpretation empirisch zurzeit beobachtbarer Vorgänge von größter Bedeutung. Das verweist natürlich auf den synthetischen Marxismus.

\section{Makroökonomischer Marxismus}

War die sektorale Betrachtungsweise eines Landes, die Diversität und Verflochtenheit der ökonomischen Aktivitäten im Fokus der Diskussion der Waren und ihrer Preise, so steht im Widerspruch dazu die politische Geschlossenheit der Nation, die sich letztlich in politisch beeinflussbaren aggregierten Größen mit denen die politische Ökonomie beschrieben wird, im Mittelpunkt des makroökonomischen Marxismus. Vieles wird dabei klarerweise von Keynes theoretischer Vision übernommen und im Sinne von Marx' Ideen modifiziert.

\footnotetext{
20 So gibt etwa (Burns 2017) einen dogmentheoretisch interessanten Einblick, der aber über den Versuch der Rekonstruktion Marxschen Denkens nicht hinauskommt.

21 Siehe (Kliman 2007) und (Freeman et al. 2004).
} 
Keynes Theorie selbst ist am besten als Reaktion auf die Unzulänglichkeiten des klassischen Marginalismus angesichts der großen Depression der 30er Jahre zu verstehen. Wenn ökonomische Theorie nicht imstande ist wirtschaftliche Katastrophen derartigen Ausmaßes auch nur ansatzweise zu erklären, so verfehlt sie eine Grundvoraussetzung jeder Theoriebildung, sie wird zu reinem Wunschdenken ohne Realitätsbezug ${ }^{22}$. Der Neuanfang, den die Makroökonomie gemäß Keynes setzt besteht darin sofort mit Variablen zu beginnen, die sich auf nationaler Ebene befinden. Für ihre quantitative Erfassung unterstützt Keynes die Einrichtung nationaler statistischer Ämter. Liegen diese nationalen Daten vor, so besteht die Aufgabe des Makroökonomen darin ihre Zusammenhänge zu beschreiben. Einige dieser Zusammenhänge ergeben sich aus der Berechnungsweise selbst, so ist etwa in einem geschlossenen System von Stromgrößen eine Größe aus allen anderen errechenbar. Was andererseits, tritt empirisch das Gegenteil auf, zur genaueren Betrachtung der zugeordneten Bestandsgrößen - alles stets auf aggregiertem Niveau - führen muss. Geht man beim Aggregieren notwendigerweise von der Heterogenität physischer Waren ab, so betreibt man Makroökonomie darüber hinaus stets als monetäre Ökonomie. Die Rückführung auf physische Größen bleibt eine vage zusätzliche Fingerübung. Statt dem Verhalten des einzelnen physischen Menschen bestimmte angeborene Gesetze zu unterstellen - den berüchtigten homo oeconomicus - geht Keynes von großen Bevölkerungsgruppen aus, deren wirtschaftliches Verhalten zumindest für einige Jahre mit sogenannten sozialpsychologischen Konstanten beschrieben werden kann. Dazu bringt er eine neue Hilfswissenschaft ins Spiel, die Ökonometrie. Hat man aber einmal aggregierte Akteure, so liegt es nahe auch den politischen Akteur Staat - den „Geschäftsführer der herrschenden Klasse“, wie Marx ihn einmal genannt hat - in das Drama des Kapitalismus einzubauen. Die Weltwirtschaftskrise hatte Keynes gezeigt, dass der Kapitalismus zu großer Instabilität neigt. Sein Fazit besteht darin die Politik wieder in die ökonomische Theorie hereinzuholen, sie wieder zu politischer Ökonomie umzubauen indem er dem Akteur Staat eine wichtige stabilisierende Rolle zuweist.

Die Rechtfertigung von Keynes' neuer Methode ist profunder als seine mit Beginn der 80er Jahre massiv intervenierenden Kritiker glauben. Die Rückführung der Bewegungsgesetze des Kapitalismus, wie sie etwa in Hicks Modell (das Keynes' Ideen formalisiert) beschrieben sind, auf die Bewegungsgesetze kleinerer, mikroökonomischer Entitäten ist für die Brauchbarkeit der makroökonomischen Theorie keineswegs ausschlaggebend. Zu Keynes Zeiten lieferte gerade die allseits bewunderte theoretische Physik das schlagende Beispiel dafür: Obwohl bereits klar wurde, dass Newtons mechanische Gesetze nur durch Durchschnittsbildung über die quer zu ihnen liegende Quantenmechanik wirklich zu Gesetzmäßigkeiten wurden, wäre niemand darauf gekommen Newtons Mechanik Sinnhaftigkeit und praktischen Nutzen abzusprechen.

\footnotetext{
22 Schlimmer noch: Sie wird zu einem ideologischen Ablenkungsmanöver, dem auch die eigenen Propagandisten aufsitzen. Keynes beschreibt das drastisch in Kapitel 12 seiner „Allgemeinen Theorie“ (Keynes 1936).
} 
Die Rückkehr der politischen Ökonomie in den Mainstream pro-kapitalistischer ökonomischer Theorie war historisch begleitet durch eine teilweise Öffnung des Staates für Einflüsse der Arbeiterbewegung - zumindest in einigen Staaten Westeuropas ${ }^{23}$. Der Staat schien zur Bühne einer friedlichen Art des Klassenkampfes zu werden und makroökonomischer Marxismus entstand als Versuch einige der Marxschen Gedanken in Keynes' Weltsicht hinüberzuretten. Die im Anschluss an die Kulturrevolte von 1968 entstandene neue Linke entdeckte Keynes für sich neu; ihn radikaler zu interpretieren als er tatsächlich war schien als Gegenmaßnahme gegen die neu erstarkten neoklassischen Autoren, die ihn zu ihrem Hauptfeind erklärt hatten ${ }^{24}$, durchaus opportun.

Eine der frühen und interessanten Arbeiten zum makroökonomischen Marxismus stammt von Stephen Marglin (Marglin 1984). Darin wird rasch klar mit welcher Art von Antagonismus sich diese Spielart des Neomarxismus hauptsächlich beschäftigt. In der Tat ist dieser schon bei Keynes Konzeption des Kreislaufes der Stromgrößen angelegt: Einerseits sollen Arbeiter so wenig wie möglich verdienen um möglichst hohe Gewinne entstehen zu lassen, andererseits kann aber gerade dadurch Überproduktion auftreten ${ }^{25}$, da die immer kleiner, wenn auch reicher werdende oligopolistische Kapitalistenklasse zu wenig Kaufkraft aufbringt. Eine solche Schrumpfungsspirale kann noch verstärkt werden, wenn gerade die Kapitalistenklasse eine höhere sozialpsychologisch erklärte Sparneigung als die Arbeiterklasse besitzt. Da bei Keynes auch die aggregierten Gesamtinvestitionen durch eine Verhaltensfunktion der Unternehmerschaft ${ }^{26}$ beschrieben werden, kann die Ersparnis sehr wohl größer sein als das Investitionsvolumen, also der Nachfrage nach Investitionsgütern. Der Widerspruch der fehlenden Nachfrage für das erzeugte Angebot stellt sich dann als Widerspruch zwischen Konsum (inklusive Investitionsnachfrage) und Sparen dar.

Geht man vom einfachen Korn-Modell aus, so ist der Widerspruch wie folgt darstellbar. Sei $\mathrm{p}$ der Preis von Korn, $\mathrm{m}$ der Lohn der Arbeit, $\mathrm{r}$ die Profitrate und $\mathrm{a}_{0}$ und $\mathrm{a}_{1}$ die Parameter der herrschenden Technologie. Wird die Output Menge auf 1 normiert und der Lohn nach der Ernte ausgezahlt, so kann die produzierte Menge

\footnotetext{
${ }^{23}$ In der Zwischenkriegszeit hatte diese Öffnung zugleich die Absicht einer Vermeidung der radikalen kommunistischen Machtübernahme nach dem Vorbild der Sowjetunion. Und zwar die Absicht sowohl der pro-kapitalistischen Kräfte als auch die Absicht der sich von den Kommunisten absetzenden Sozialdemokraten Europas.

${ }^{24}$ Als politische Devise hatten die konservativen Regierungen dieser Zeit die Zurückdrängung staatlichen Einflusses ausgegeben. De facto war damit jedoch nur die Eliminierung der sozialstaatlichen Dimension, also des Einflusses der Arbeiterbewegung gemeint. Der Staat sollte wieder zum alleinigen Instrument der herrschenden Unternehmerschaft werden und wie Firmen traten nun ganze Staaten in den ,internationalen Wettbewerb" ein. Ein großer Teil der sozialdemokratischen Führung folgte denn auch dem neuen Credo und betrieb - unter Beibehaltung ihrer Regierungsämter - Profitmaximierung auf staatlicher Ebene, waren also funktional Teil der herrschenden Klasse.

${ }^{25}$ Diese Form der Überproduktionskrise hatten Marx und andere Autoren tatsächlich bereits im vorhergehenden Jahrhundert beschrieben.

${ }^{26}$ Die Sache wird wesentlich komplizierter wenn Erwartungsprozesse in der Investitionsfunktion eingebaut werden.
} 
zum Teil den Arbeitern zum Konsum verkauft und zum Teil für die neue Aussaat verwendet werden ${ }^{27}$ :

$$
\mathrm{p}=\mathrm{m} \cdot a_{0}+(1+\mathrm{r}) \cdot \mathrm{p} \cdot a_{1}
$$

Definiert man den Reallohn w als Quotienten von m und p so kann diese Verteilungsgleichung folgendermaßen umgeformt werden:

$$
w=\frac{1-a_{1}}{a_{0}}-\frac{a_{1}}{a_{0}} \cdot r
$$

Der erste Term ist das Nettoprodukt pro Arbeitseinheit, der zweite Term beschreibt die durch die Technologie modifizierte Profitrate.

Ähnlich kann der Output nach seiner Verwendung beschrieben werden. Ist c die Konsummenge, die die Arbeiter zu ihrer Reproduktion benötigen und g die Wachstumsrate der Kornwirtschaft so muss für diese Quantitäten gelten:

$$
c \cdot a_{0}+(1+g) \cdot a_{1}=1
$$

Durch einfache Umformung erhält man

$$
c=\frac{1-a_{1}}{a_{0}}-\frac{a_{1}}{a_{0}} \cdot g
$$

Die beiden Gleichungen können in einem Diagramm zusammengefasst werden, der Winkel k beschreibt die durch den Quotienten aus $\mathrm{a}_{1}$ und $\mathrm{a}_{0}$ beschriebene Kapitalintensität.

Marx Argumentation fließt insofern in das Modell ein als die Kapitalistenklasse gezwungen ist den für die Reproduktion der Arbeiter gerade noch möglichen geringsten Lohn, $\mathrm{w}_{0}$, zu zahlen, nur so können sie ihre Profirate, $\mathrm{r}_{0}$, maximieren. Für die Seite der Arbeiterklasse garantiert eine genügend hohe Arbeitslosenrate, dass sie diesen Subsistenzlohn auch akzeptiert. Die eingetragenen Pfeile in Abb. 1 sollen zeigen, dass damit auch das größtmögliche Wachstum der Kornwirtschaft erzielt wird.

Wächst die Kornwirtschaft so wird es auch möglich mehr Arbeiter zu beschäftigen, damit aber das hohe Wachstum via Subsistenzlohn erhalten werden kann muss die Arbeitslosenrate hoch bleiben, am besten indem das Arbeitsangebot schneller wächst. Hier berührt die Analyse des makroökonomischen Marxismus jene der konservativen Ökonomen unserer Tage: Um Wirtschaftswachstum zu erzeugen muss der heutige Konsum minimiert werden und das Arbeitsangebot (etwa durch späteres Pensionsalter) erhöht werden. Der Unterschied in der Interpretation kommt in erster Linie durch das Fehlen des Klassenaspekts bei Letzteren zustande. Zerfällt die Gesellschaft in Klassen bei denen die eine durch Subsistenzlohn und Arbeitslosig-

\footnotetext{
27 Hier wird von einem konstanten Preisniveau ausgegangen, das heißt Preise dienen nur zur Korrektur für wetterbedingte Schwankungen des Ernteerfolgs. Da dieser langfristig einen konstanten Mittelwert habe stelle sich ein sogenannter ,natürlicher“ Preis ein. Das folgt der klassischen ökonomischen Literatur des 19. Jahrhunderts.
} 
Abb. 1 Das Korn-Modell

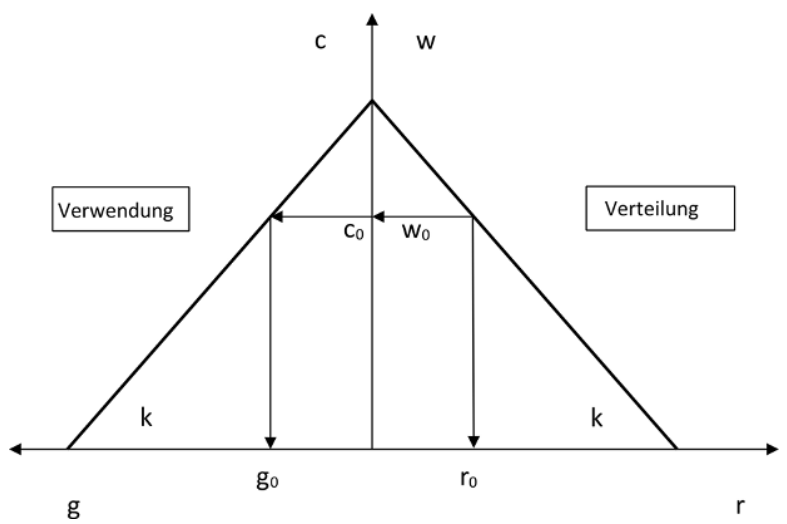

keit gekennzeichnet ist, während die andere einem schrankenlosen Wachstumsdiktat gemäß handelt, so ist eine explosive Entladung dieses Klassenwiderspruchs unausweichlich. Bezüglich der Auflösung des Widerspruchs bleibt der makroökonomische Marxismus allerdings genauso stumm wie die konservative Interpretation, die ihn per Annahme eliminiert ${ }^{28}$.

Ein weiterer Pferdefuß dieses Ansatzes besteht offensichtlich darin, dass das wichtigste Argument für das historische Auftreten des Kapitalismus, das Element, das Marx für die „historische Mission“ des Kapitalismus hält, fehlt: Die Entwicklung technischen Fortschritts. Über die Veränderung der beiden Parameter, die diesen im Kornmodell beschreiben, wird keine Aussage getroffen. Selbst in der viel breiteren Diskussion innerhalb des Mainstreams wird mit „endogenem technischen Fortschritt" meistens nur der trade-off zwischen der Produktion von sofort konsumierbarem „Korn“ und umschichtbaren Einsatz von Arbeitszeit für bessere Produktivitätsparameter verstanden, also wiederum ein Widerspruch bloß zwischen heute oder morgen mehr konsumieren. Recht paradox wird diese Diskussion, wenn sich der immer noch virulente methodische Individualismus einmischt und Klassenwiderspruch als gegensätzliche Zeitpräferenzraten in der Bevölkerung stilisiert werden ${ }^{29}$. Die Wachstumsprotagonisten sind dann die braven Sparer, denen künftiger Konsum lieber ist als momentaner Konsum, während diejenigen die eine hohe Zeitpräferenz haben zur Klasse der Konsumsüchtigen werden.

Ein besonders interessanter Fall von makroökonomischem Marxismus ist das Grundmodell von Richard Goodwin (Goodwin 1967). Hier fällt technischer Fortschritt zwar ebenfalls nur wie „Manna vom Himmel“, es wird aber zumindest gezeigt wie kontinuierliches Wachstum mit zyklischen Bewegungen von Verteilung und Arbeitslosigkeit kombiniert werden kann. Um das zu ermöglichen musste Goodwin über die zumeist linearen Modelle der Keynesianer hinausgehen und ein der Biolo-

\footnotetext{
28 So wird typischerweise angenommen, dass jede Person in gleichem Ausmaß zugleich Arbeiter und Kapitalist ist, es also nur eine Klasse gibt. Die Abwägung zwischen heutigem Konsum und späteren Konsum ist dann einzig eine Frage der Zeitpräferenz dieses unterstellten homo economicus. Für die wirtschaftspolitische Diskussion kann sie dann nur mehr für eine oberflächliche Diskussion des Generationenkonflikts herhalten.

29 Frank Ramsey hat den entsprechenden Formalismus sehr früh schon in seine Nutzenfunktion eingebaut (Ramsey 1928).
} 
gie entlehntes nichtlineares Modell, das Lotka-Volterra Modell, für die Ökonomie umdeuten. Darin finden sich Spurenelemente marxistischen Ursprungs: (1) Die Arbeitslosigkeit spielt eine essentielle Rolle indem sie die Lohnbildung beeinflusst; (2) das Modell ist dynamisch instabil, das heißt jede Parameterverschiebung führt zu andersartigen Konjunkturzyklen ${ }^{30}$; (3) eine Zuordnung der Verhaltensgleichungen zu bestimmten Klassen ist möglich.

Im letzten Punkt gleicht Goodwin damit anderen von Marx beeinflussten Keynesianern, die ebenfalls versuchten den makroökonomischen Verhaltensfunktionen bestimmte gesellschaftliche Klassen zuzuschreiben. Kaldor zerlegte die Konsumfunktion in einen Teil für Arbeiter und einen Teil für Kapitalisten (Kaldor 1955), Kalecki (Kalecki 1942) und andere arbeiteten intensiv an der Gestaltung der Investitionsfunktion als einer die Kapitalistenklasse beschreibenden Funktion. Insofern hier neue Elemente zu Keynes Modell hereingenommen wurden kann manchmal von einer marxistischen Beeinflussung der sogenannten post-keynesianischen Theorie gesprochen werden. In aller Regel verstehen sich die Vertreter dieser Ansätze selbst zurecht nicht als neomarxistische Ökonomen.

\section{Evolutionstheoretischer Marxismus}

Eine etwas schwächer erforschte Seite von Marx Arbeiten ist seine Affinität zur Evolutionstheorie. Schon in seinen jungen Jahren als Schüler Hegels war er zweifellos davon überzeugt, dass die Menschheitsgeschichte eine in Perioden unterteilbare Sequenz aufweist. Hegel war - als deutscher Repräsentant der Ideen der französischen Aufklärung - zwar immer noch der Auffassung alle Dialektik bewege sich zu einem Absoluten hin, das in seiner Omnipräsenz durchaus auch als Gott bezeichnet werden sollte, er betonte aber zugleich, dass Negation stets auch bestimmte Negation, Aufhebung eines besonderen Zustands sei. Die Abfolge der besonderen Negationen zu studieren sei eben genau die Aufgabe der Wissenschaft. Beim Nachzeichnen der geschichtlichen und wissenschaftlichen Epochen war es aber unmöglich nicht auch entlang der führenden Persönlichkeiten der jeweiligen Ära zu argumentieren. Implizit war daher immer auch mitgedacht, dass der Durchbruch zu einer neuen Epoche von besonderen einzelnen Trägern des Fortschritts, von Pionieren, die die kulminierenden Widersprüche der untergehenden Ära in eine neue vermittelbare Vision ummünzen konnten initiiert wurde.

Marx selbst sah sich sein Leben lang als genau eine dieser besonderen Persönlichkeiten, die eine Revolutionierung der herrschenden Produktionsweise in Gang bringen könnte. Seiner Einschätzung nach hätte die bürgerliche Revolution von 1848 unmittelbar in die proletarische Revolution münden sollen. Die erstere versandete im aufgeklärten Absolutismus, die zweite fand nicht statt. Der grundlegende Wider-

\footnotetext{
30 Dadurch können solche Verschiebungen leicht endogenisiert werden, womit explodierende Zyklen leicht modellierbar werden. In der Tat genügt es schon das ursprünglich in kontinuierlicher Zeit formulierte Modell in diskrete Zeit umzuformulieren um explodierende Zyklen zu modellieren. Damit wird Marx Idee der Unausweichlichkeit von Revolutionen angesprochen, auch wenn die revolutionäre Umstrukturierung nach wie vor im Dunkeln bleibt.
} 
spruch mit dem sich evolutionärer Marxismus befasst ist bereits an diesem biographischen Detail ablesbar: Die innovatorische Anstrengung einzelner Entitäten sind mit dem Risiko des Scheiterns behaftet. Einerseits müssen sie an den Erfolg ihrer Mission glauben um die Energie für die Überwindung der sich ihnen entgegenstellenden Traditionen aufzubringen, andererseits gebietet ihnen aber die Notwendigkeit ihr kurzfristiges Überleben zu sichern, ein klarsichtiges Einschätzen der momentanen Möglichkeiten zu kultivieren. Machbares und Visionäres müssen parallel betrieben werden. Marx' diesbezügliche Taktik bestand meist darin das Visionäre nur ganz strikt als Lösung der momentan sichtbarsten Widersprüche des Kapitalismus seiner Zeit zu formulieren. Koalitionen und Streit zwischen den sozialrevolutionären Führungspersönlichkeiten waren im 19. Jahrhundert an der Tagesordnung. Als letztlich der 1. Weltkrieg das Ende der meisten feudalen Systeme brachte waren die Nachfolgeregime auch durch deren ideologische Prägungen mitbestimmt.

Die Metapher vom Durchbruch neuer Ideen, neuer Technologien, neuer institutioneller Verflechtungen hat ein sehr konkretes Vorbild in der biologischen Evolutionstheorie Darwins (Darwin 1872). Bestimmte Arten mit charakteristischen Eigenschaften setzen sich durch wenn genau diese Eigenschaften besonders gutes Überleben in einer lange Zeit konstant bleibenden Umgebung ermöglichen. Wenn aber schließlich (eventuell gerade durch den Erfolg dieser Spezies) die Umweltbedingungen sich ändern und einer neuen Spezies bessere Chancen einräumen, so werden auch zunächst einzelne Pioniere als mögliche Kandidaten verschiedener neuer Arten auftreten. Einige werden scheitern bis sich schließlich eine neue Konstellation durchsetzt.

Marx Zitat von den Revolutionen als den Schnellzügen der Geschichte bezieht sich offenbar auf die relativ hohe Geschwindigkeit mit der sich ein solcher Strukturwandel in menschlichen Gesellschaften im Gegensatz zur Biologie vollzieht. In der neueren marxistischen Literatur ist der Gedankengang an unterschiedlichen Stellen eingegangen.

Bei den Ökonomen rund um David Gordon ${ }^{31}$ wurde und wird an der New School for Social Research am Konzept der Social Strata of Accumulation (SSA) gearbeitet. Darunter wird ein ökonomisches und institutionelles Arrangement verstanden, das über einen längeren Zeitraum hinweg stabile Kapitalakkumulation gewährleistet. Die von Marx eingeführte Periodisierung der Geschichte in Produktionsweisen wird also weiter verfeinert indem etwa die Produktionsweise Kapitalismus in mehrere SSA gegliedert wieder. In ähnlicher Weise wurde sie in (Hanappi 1986, 2018) in die drei Stufen - Handelskapitalismus, Industriekapitalismus, integrierter Kapitalismus - gegliedert. Gordons SSA beziehen sich allerdings stärker auf nationales institutionelles Rahmenwerk und sind daher eine feinere Gliederung (genauer Bezug nehmend auf die territoriale Ausbreitung des Kapitalismus) als die drei erwähnten Stufen. Das Spannungsfeld des Widerspruches zwischen dem visionären Entwurf und dem mühseligen Alltag der kleinen Verbesserungen des sozialen Lebens kommt besonders gut in einer Gegenüberstellung der Politikvorschläge in (Gordon et al. 1985) und den postulierten Ansprüchen des Egalitarismus zum Ausdruck. Während die für die USA formulierten wirtschaftspolitischen Vorschläge nirgends über das

31 Siehe (Gordon 1978). 
hinausgehen was in europäischen Staaten, insbesondere in Skandinavien, bereits verwirklicht wurde, bleiben die egalitären Wunschvorstellungen meist in recht vagen Formulierungen und allgemeiner Kritik imperialistischer Politik verhaftet. Viele der als mögliche visionäre Pionierleistung anvisierten Ansätze angelsächsischer Autoren in diesem Umfeld sind dadurch charakterisierbar, dass sie einerseits tief im neoklassischen Handwerkzeug der Modellierung verhaftet sind (Gleichgewicht, Nutzenfunktionen, Optimierung) und andererseits auch keinen Bezug zum methodischen Hintergrund (Dialektik) der Arbeit in Marxscher Tradition haben oder herstellen wollen $^{32}$. Es mag sein, dass der Zugang zu diesem Aspekt auch durch die enge sprachliche Verknüpfung dialektischen Denkens mit den Eigenheiten der deutschen Sprache angelsächsischen Autoren besonders schwerfällt ${ }^{33}$.

Innovative Pioniere, dort Unternehmer genannt, waren auch das zentrale Thema Joseph Schumpeters. Das Tätigkeitsfeld dieser Pioniere war allerdings typischerweise nicht die Herstellung neuer gesellschaftlicher Produktionsverhältnisse oder SSA, sondern vielmehr die Einführung neuer Produktionsmethoden oder Produkte in einem ganz spezifischen Teilbereich der Produktion (Schumpeter 1911). Ausschlaggebend für den offensichtlichen Erfolg der ,historischen Mission“ des Kapitalismus, der enormen Steigerung der Arbeitsproduktivität, war für ihn das riskante Erproben neuer Kombinationen bestehender Erfindungen und Techniken im gesellschaftlichen Umfeld $^{34}$. Bei Marx war dieser Prozess in der Annahme des steten Wachstums der Produktivkräfte versteckt, Schumpeters Forschungsinteresse galt genau dem Ausfüllen dieser Lücke. Schumpeter selbst war, obwohl er im Gegensatz zu Keynes Marx genau gelesen hatte, weit davon entfernt sich als Marxist zu verstehen. Er gestand jedoch zu, dass er wie Marx von Tatsachenbeobachtungen ausgehe und diesbezüglich mit ihm methodisch übereinstimme. Wie Marx sah auch er den Kapitalismus nicht als endgültiges Stadium der Menschheitsentwicklung, sondern als enden wollende Epoche an. Und zwar werde sie genau dann enden, wenn das Wachstum der Produktivkräfte, die innovative Kraft der Unternehmer, versiegt. Der danach eintretenden Produktionsweise sah Schumpeter allerdings wenig euphorisch entgegen. Seine Vision des „Sozialismus“" war ein von Verwaltungstechniken dominierter grauer Alltag in dem vor allem die von ihm farbenfroh heroisierte Figur des Unternehmensführers fehlte. Im Prinzip teilte er diese Einschätzung mit Friedrich Engels, der ebenfalls eine Dominanz verwaltungstechnischer Vorgänge vorhersagte, wenn auch mit deutlich freudigerer Grundstimmung. Marx selbst hat sich in Bezug auf Visionen sehr zurückgehalten ${ }^{35}$. Nach 1848 wurde er von seiner eigenen dialektischen Projektion der revolutionären Rolle auf das Proletariat hinweg getragen - und als diese nicht

\footnotetext{
${ }^{32}$ Ganz eklatant sichtbar etwa bei der Schule des Analytischen Marxismus (Roemer 1981). Eine Ausnahme stellt diesbezüglich Duncan Foley dar, der trotz hervorragender Beherrschung neoklassischer Methoden stets um methodische Innovation bemüht ist (Foley 1995).

${ }^{33}$ Französische Autoren sind zwar durch die Geradlinigkeit ihrer Sprache ebenfalls gehandicapt, haben diese Schwierigkeit aber durch die Erfindung eines eigenen französischen Sprachspiels (im Sinne Wittgensteins) in langer Tradition überwunden, siehe (Merlau-Ponty 1955).

${ }^{34}$ Dass damit auch die Finanzierung solcher Innovationen ein essentielles Thema war macht klar, dass Schumpeter - wie Marx - auch zu den monetär argumentierenden Ökonomen zu zählen ist.

${ }^{35}$ Das „Jeder nach seinen Fähigkeiten, jedem nach seinen Bedürfnissen.“ (Marx 1874) ist wohl das Konkreteste, das aus seinen Schriften herauszulesen ist.
} 
eintrat, trat er selbst den Rückzug in genaueres Studium der politischen Ökonomie und Vertiefung seiner theoretischen Arbeit an. Auch Schumpeter hat als letztes Werk eine Dogmengeschichte verfasst (Schumpeter 1954).

Angesichts der Ähnlichkeiten wurde Schumpeter manchmal als der „Marx der Bourgeoisie“" bezeichnet (Catephores 1994); sein wichtigster Beitrag, nämlich seine Innovationstheorie, ist aber eher als ein zu Marx komplementäres Theoriefragment $\mathrm{zu}$ verstehen. Zwei Merkmale sind besonders hervorzuheben: (1) Innovation geschieht nicht an allen Stellen des Systems auf einmal, sondern wird durch eine kleine Menge diverser Entwicklungsvorschläge in unterschiedlichen Systembereichen initiiert; (2) auf die erfolgreiche Initiierung einiger weniger (viele scheitern) erfolgt sehr rasch ein Schwärmen anderer Innovationen durch die Implikationen dieser Erfolge. Offensichtlich folgt auch Schumpeter hier der methodischen Maxime Widersprüchliches zu beschreiben: Einerseits wird die Vereinzelung des Innovationsvorganges betont - er findet auf betrieblicher Ebene statt - andererseits wird die rasche Übertragung innovativen Erfolgs auf die Gesamtwirtschaft hervorgehoben.

Schumpeter selbst hat, wie Keynes, selbst nie ein formales Modell seiner Theorie vorgelegt. Sein Assistent in Harvard, Richard Goodwin, hat das aber sehr wohl getan und hat technischen Fortschritt in sein eigenes Modell des oszillierenden Kapitalismus eingebaut (Goodwin 1990). In diesem Modell werden die kürzeren Konjunkturzyklen (Juglar-Zyklen) durch langfristige Zyklen von Basisinnovationen (Kondratieff-Zyklen) überlagert. Dieses Modell wäre wohl als ein Hybrid zwischen makroökonomischem und evolutionstheoretischen Marxismus einzuordnen ${ }^{36}$, da auch dort technischer Fortschritt nur sehr aggregiert vorkommt. Eine ausführlichere Behandlung unter Verwendung von I-O Techniken findet sich schließlich in (Goodwin und Punzo 1987).

Eine Reihe neuerer Autoren haben die Tradition der Schumpeter-Goodwin Schule fortgesetzt, manche mit stärkerer Einbeziehung des keynesianischen Einflusses auf Goodwin ${ }^{37}$, manche mit stärkerer Betonung des innovativen Zufalls wie bei Schumpeter ${ }^{38}$. Eine explizite Einbeziehung von Erwartungsbildungsprozessen die zu einem evolutionstheoretischen Lotka-Volterra System à la Goodwin führen findet sich bei (Hanappi 2006).

In älteren sozialwissenschaftlichen Debatten wurde Evolution und Revolution üblicherweise als Gegensatzpaar betrachtet. Eine Verbindung einer evolutionären Entwicklung mit einer am exponiertesten Vertreter revolutionären Wandels, Karl Marx, orientierten Denkschule scheint deshalb nicht nur widersprüchlich, sondern geradezu unmöglich zu sein. Bei genauerer Betrachtung von Evolution, insbesondere auch biologischer Evolution, zeigt sich aber, dass sie in einander abwechselnden Phasen von relativ langsamer Anpassung und relativ kurzen Zeiträumen revolutionären

\footnotetext{
36 Zum Verhältnis zwischen Schumpeter und Goodwin siehe (Hanappi 2015).

37 Peter Flaschel etwa stellt seine Modellierungen explizit als Marx-Keynes-Schumpeter Modelle vor (Flaschel et al. 2018). Wie Marx sieht auch er die Notwendigkeit des kurzfristigen wirtschaftspolitischen Eingriffs und schreckt daher - glücklicherweise und leider durchaus unüblich unter Theoretikern - vor konkreten Verbesserungsvorschlägen nicht zurück (Flaschel und Luchtenberg 2012).

38 Giovanni Dosi hat ein sehr einflussreiches Innovationsmodell vorgelegt (Dosi 1982) und seine Arbeitsgruppe hat sich in den letzten Jahren verstärkt und sehr erfolgreich mit der Simulation von Innovationsverhalten beschäftigt.
} 
Strukturwandels ${ }^{39}$ verläuft. Revolutionen sind so gesehen Teilprozesse der Evolution. Umgekehrt verläuft bei genauerer Betrachtung auch jede Revolution zeitlich, wenn auch in oft kurzen Phasen $\mathrm{ab}^{40}$. Jede dieser kurzen Phasen ist als Evolution zwischen Brüchen beschreibbar, Revolutionen beinhalten also Evolution. Eines der Mysterien des in diesem Kapitel behandelten Widerspruchs besteht genau darin die Verhaltensweise der innovativen Pioniere (in Gesellschaft und Theorie) angesichts der unterschiedlichen Fristigkeiten von Revolution und Evolution zu verstehen. Evolutionstheoretischer Marxismus geht dem nach was Rosanna Rosanda einmal als Dialektik von Bruch und Kontinuität bezeichnet hat, siehe (Rosanda 1974) und berücksichtigt dabei insbesondere die Vorreiterrolle einzelner innovativer Revolutionäre. Wie kommt das Neue in die Welt? Im Übersichtsartikel „Evolutionary Political Economy: Content and Method“ zeigen Hanappi und Scholz-Wäckerle auf wie dieser Ansatz in den größeren Strom evolutionärer Theorie eingebettet werden kann (Hanappi und Scholz-Wäckerle 2017).

Es ist verständlich, dass ein guter Teil der Forscherinnen und Forscher in diesem Bereich sich sehr weit von dem was gemeinhin als Marxismus verstanden wird entfernt sehen. Besonders hier ist Marx eigener Beitrag zur Entwicklung dieser Widersprüche sehr gering. Es ist auch gar nicht nötig sich zu einer Spielart des Marxismus zu bekennen. Der Einfluss geschichtswirksamer Denkschulen ist anders als ein Religionsbekenntnis stets in Richtung Zukunft orientiert ${ }^{41}$, was und von wem auch immer an vergangenem Wissen brauchbar verwendet werden kann ist wertvoll. Die Verbreiterung eines erfolgreichen Ansatzes auf benachbarte wissenschaftliche Disziplinen ist gerade wegen des Erfolgs unausweichlich. Wenn dabei die eine oder andere Etikettierung verloren geht so ist das in der Regel nicht tragisch. Der nächste neomarxistische Strom ist daher noch stärker als „neo-marxistisch“ zu verstehen.

\section{Synthetischer Marxismus}

Marx wurde 1818, vor 200 Jahren, geboren und starb 1883. Keynes und Schumpeter wurden beide in Marx Todesjahr 1883 geboren. Kurz davor, 1874, entstand die marginalistische Theorie (Walras, Jevons, Menger) auf der bis heute die Standardökonomie aufbaut. Sie war nicht zuletzt eine Antwort auf die klassische politische Ökonomie, insbesondere auch auf Marx, indem sie politische Neutralität vortäuschte aber dennoch anti-feudal, weil marktorientiert war. In den späten 40er Jahren baute Paul Samuelson, Assistent Schumpeters in Harvard, diese Theorie in die mathematisch formulierte neoklassische Synthese aus, die bis heute als Grundlage der Mikroökonomie gilt ${ }^{42}$. Seit den späten 30er Jahren hatten Keynes' Epigonen auch

\footnotetext{
${ }^{39}$ Bei „Strukturwandel“ ist hier stets zu beachten, dass darunter nicht nur eine generelle Veränderung der Beziehungen zwischen den Strukturelementen verstanden wird, sondern auch das Verschwinden und Entstehen neuer Elemente zu inkludieren ist.

${ }^{40}$ Man vergleiche Marx minutiöse Studie der 1848 Revolution im „18. Brumaire des Louis Bonaparte“ (Marx 1852).

${ }^{41}$ So wollte Schumpeter nie als evolutionärer Ökonom bezeichnet werden und Marx hatte seine Probleme mit dem Etikett Marxismus, etc.

42 Siehe (Samuelson 1947).
} 
dessen Ideen zur mathematisch formulierten Makroökonomie ausgebaut, e.g. (Hicks 1937). Daneben schwelten all die Zeit die Reste des Marxschen Gedankenguts. Ihre Verwandlung in eine Staatsreligion in der Sowjetunion nach dem 1. Weltkrieg hatte Folgen. Einerseits war Marx Werk dadurch in die ganze Welt verbreitet worden. Andererseits war mit dem raschen Übergang zum Stalinismus ab 1924 eine höchst verkommene Form der Interpretation zum ideologischen Repressionsmittel geworden ${ }^{43}$. Das hat denn auch das Wort „Marxismus“, besonders in den stalinistisch regierten Staaten nachhaltig desavouiert. Erst im Anschluss an die Jugendrevolte von 1968 erlebten Marx' Werke ein erneutes kurzes Aufflackern. Das wurde allerdings durch ein recht rasches Verschließen akademischer Karrieren einerseits und die Aufgabe der Unterstützung Marxscher Ideen durch sozialdemokratische Parteien - diese hatten sich stattdessen dem Keynesianismus als Katechismus verschrieben - schnell zum Erliegen gebracht. Doch im Verborgenen glühte das Flämmchen weiter.

Nach dem Zusammenbruch der Sowjetunion schien es als ob von den drei dominierenden Gesellschaftssystemen des 20. Jahrhunderts - Kapitalismus, Sozialismus und Faschismus - nur mehr der Kapitalismus als siegreiches System übriggeblieben wäre. Doch der Schein währte nur kurz. Im Vakuum der großen Reiche Eurasiens entstand kein natürlicher, als bestes System davor nur gewaltsam von stalinistischen Cliquen unterdrückter Kapitalismus. Damit kollabierte auch die Hoffnung westlichen Kapitals sich hier flächendeckend ein prosperierendes neues Betätigungsfeld zu eröffnen ${ }^{44}$. Während in Westeuropa die Sozialdemokratie mit ihrem Motto „Kapitalismus mit menschlichem Antlitz" ihre Aufgabe als ideologische Abwehr gegen stalinistische Regime zunehmend verlor und aus Regierungen verdrängt wurde, entstanden im Osten neue autokratische Reiche, deren Motto ,Sozialismus mit kapitalistischem Antlitz" heißen könnte. Eine straffe politische Struktur - Erbe des klassischen Stalinismus - gepaart mit längerfristig planbaren Algorithmen kapitalistischer Akkumulation einer herrschenden Elite haben sich in den letzten beiden Jahrzehnten als außergewöhnlich tragfähiges Konzept herausgestellt. Inwieweit Kapitalismus damit in einer neuen Produktionsweise aufgehoben wird sei dahingestellt. Es ist jedoch bemerkenswert wie der Kapitalismus in den USA, durch seine Krisen von 2001 und 2008 schwer getroffen, sich in Formen wandelt, die sich dieser neuen autokratischen Form annähern. Für Europa stellt sich die Frage ob es dem in Form der neuen Autokratien auftretenden neuen Gesellschaftsmodell durch seinen Einigungsprozess ein demokratischeres Modell entgegenstellen kann - oder ob es zu einem zwischen den Blöcken zerriebenen, in neo-koloniale nationalistische Kleinstaaten zerfallendes Konglomerat zurückfällt.

Im Kontext dieser dramatischen politischen Dynamik stellt sich die Frage inwieweit Marx' Ansätze überhaupt noch zu einer für die politische Praxis relevanten theoretischen Alternative werden können. Sind sie nicht mit dem nun mehr oder weniger endgültigen Verschwinden des klassischen Industriekapitalismus seiner Zeit als genau dessen Kritik ebenso obsolet wie dieser? Die Protagonisten eines synthetischen

\footnotetext{
${ }^{43}$ In gewisser Weise ist die Übernahme des Christentums zur Staatsreligion des Römischen Reiches (300 nach Christus) eine historische Parallele.

${ }^{44}$ Ausnahmen gab es allerdings, insbesondere im Rahmen bestimmter Übereinkünfte mit der chinesischen Führung.
} 
Marxismus verneinen diese Frage. Und zwar durch den Verweis darauf, dass Marx zwar kein geschlossenes Theoriegebilde konstruiert hat, sich aber in methodisch konsistenter Weise als radikalster - an die Wurzel gehender - Kritiker profiliert hat. Da der Kapitalismus nun in eine seiner schwersten Krisen schlittert ist eine radikale Kritik politischer Ökonomie gefordert wie selten zuvor. Die Standardökonomie ist mit ihrer Zersplitterung in Mikro- und Makroökonomie, mit ihrer Rückkehr zu ihren strikt marginalistischen Wurzeln zu einem zahnlosen Ablenkungsmanöver verkommen. Und zwar trotz, öfter sogar wegen ihrer immer ausgefeilteren mathematischen Methoden. Die an sich zu begrüßende Inkludierung von Erwartungsprozessen blieb theoretisch folgenlos, da absurde Informationsannahmen, etwa in der „Neuen Klassischen Makroökonomie" ${ }^{\text {45 }}$ (eine eklatante Fehlbenennung) dieser Erweiterung den Stachel sofort wieder entzogen ${ }^{46}$.

Der Widerspruch, dem synthetischer Marxismus sich stellen muss - den er ausspricht - ist Folgender: Einerseits liegt eine beachtliche, sehr heterogene Menge an neomarxistisch inspirierten Theoriefragmenten in vielen Disziplinen vor ${ }^{47}$. Um diese entsprechend zu würdigen müssen Kenntnisse des jeweiligen Teilbereichs vorhanden sein, beziehungsweise erworben werden, erst dann kann Synthese beginnen. Andererseits ist rasches politikorientiertes Handeln als gesellschaftlich relevanter Akteur nötig; und zwar in einem Umfeld, in dem seinerseits die Trennlinien zwischen Politik, Ökonomie, Massenkommunikation, Massenmanipulation und deren technischen Möglichkeiten bereits verschwommen sind. Schon die erste, wissenschaftlich orientierte Seite des Widerspruchs ist keine Aufgabe, die einzelne Forscher bewältigen können, sie benötigt transdisziplinäre, globale Vernetzungen zu Teams. Diese Netzwerke sind momentan im Entstehen, brauchen aber Zeit und stoßen trotz der unendlich wertvollen Existenz des Internets auf sprachliche und (wissenschafts-) kulturelle Hindernisse. Wie schon in Marx eigenem Leben ist andererseits die Suche nach dem revolutionären Subjekt, der revolutionären Klasse, in der die Theorie wirklichkeitswirksam werden kann eine dringende und schwierige Aufgabe ${ }^{48}$. In unübersichtlichen Zeiten ist die Dynamik der globalen Klassenbildungen verstärkt auch auf Intuition angewiesen. Marx hatte seiner eigenen Geschichtsinterpretation und der Hegelschen Betonung des Arbeitsbegriffes folgend - ebenfalls mit einem gewissen Maß an Intuition ${ }^{49}$ - das Proletariat als den Helden der nächsten Revolution erwählt. Etwa 170 Jahre später ist nun davon auszugehen, dass sich die globale

\footnotetext{
45 Siehe (Sargent 1987).

${ }^{46}$ Ein ähnliches Schicksal erfuhren große Teile der Spieltheorie in denen neoklassische Vorurteile die prinzipielle Flexibilität des Ansatzes untergruben, siehe (Hanappi 2013).

47 In der Ökonomie reicht das etwa von Alan Freeman (Freeman et al. 2004) bis Elmar Altvater (Altvater 2011), in Politikwissenschaft von Nicos Poulantzas (Poulantzas 2000) bis Sarah Wagenknecht (Wagenknecht 2016), in der Soziologie von Eric Ohlin Wright (Wright 2017) bis Pierre Bourdieu (Bourdieu 1996), in der Philosophie von Slavoj Žižek (Žižek 2015) bis Alain Badiou (Badiou 2011). Von der stürmischen Entwicklung in Formalisierungstechniken, die ebenfalls in die Synthese einzubinden wäre gar nicht zu reden, siehe (Hanappi 2013, 2014, 2017).

${ }^{48}$ Gramsci hat für diese Aufgabe den Begriff des „organischen Intellektuellen“ vorgeschlagen, der einerseits die nötige intellektuelle Kapazität einbringt, andererseits aber auch in der Klassendynamik ,organisch" verankert ist (Gramsci 1999).
}

49 Siehe die Beschreibungen in (Jones 2016). 
Klassenstruktur entscheidend weiterentwickelt hat. Selbst wenn in Marx Tradition am Primat des Arbeitsbegriffes festgehalten wird, so liegt nicht auf der Hand welche Klasse(n) heute zum revolutionären Subjekt taugen. In dieser Situation erprobt synthetischer Marxismus mögliche, temporäre Koalitionsbildungen: mit grünen Bewegungen, mit Feminismus, mit anti-rassistischen Bewegungen, etc ${ }^{50}$.

Innerhalb der politischen Ökonomie stellt diese zweifache, widersprüchliche Anstrengung einen markanten Kontrast zur Standardökonomie dar. Der Synthese in der Theoriebildung steht bei der Standardökonomie die weiterhin fortschreitende, Taylor folgende Spezialisierung gegenüber. Nicht zuletzt daher rührt auch ihre Sprachlosigkeit gegenüber Multidisziplinarität verlangenden, wirtschaftspolitischen Aufgaben. Folgerichtig hat die Standardökonomie auch kein Problem damit die Reduktion ihres wirtschaftspolitischen Einflusses auf ein ideologisches Ablenkungsmanöver (auch in Bezug auf ihre eigenen Protagonisten) in falscher Bescheidenheit als „Objektivität“ zu tarnen. Es braucht keinen progressiven Motor in der Gesellschaftsentwicklung, denn der objektive Geist ökonomischer Rationalität wird schon ähnlich wie der technische Fortschritt wie „Manna vom Himmel“ fallen.

\section{Fazit}

Für die letztgenannte Kategorie neomarxistischer Ansätze, den synthetischen Marxismus, ist die Einbettung in eine Marx folgende methodische Tradition vielleicht gar nicht mehr allzu sinnvoll. Sie ist inzwischen Teil einer sich als progressiv verstehenden wissenschaftlichen Bewegung für die die Orientierung an herausragenden wissenschaftlichen Persönlichkeiten nur mehr inhaltlich, aber nicht mehr reputationsmäßig ins Gewicht fällt. Es ist das zweifellos auch immer noch das Beste was einem bedeutenden Wissenschaftler passieren kann: Wenn das präsentierte Gedankengut in so vielen Bereichen Eingang gefunden hat und zu einer Selbstverständlichkeit geworden ist mit der die praktizierenden Forscher umgehen ohne auch nur mehr zu ahnen von wem es stammt, dann hat der Gelehrte seine individuelle Rolle in vollem Umfang erfüllt und diese ist im allgemeinen Wissen aufgegangen - oder mit Hegel: aufgehoben.

Funding Open access funding provided by TU Wien (TUW).

Open Access Dieser Artikel wird unter der Creative Commons Namensnennung 4.0 International Lizenz (http://creativecommons.org/licenses/by/4.0/deed.de) veröffentlicht, welche die Nutzung, Vervielfältigung, Bearbeitung, Verbreitung und Wiedergabe in jeglichem Medium und Format erlaubt, sofern Sie den/die ursprünglichen Autor(en) und die Quelle ordnungsgemäß nennen, einen Link zur Creative Commons Lizenz beifügen und angeben, ob Änderungen vorgenommen wurden.

\footnotetext{
50 Als interessante Anstrengungen eines synthetischen Marxismus seien etwa (Gintis 2016) und (Fuchs 2016) in den Bereichen Spieltheorie und Informationswissenschaft und der in der Tradition von Adorno stehende Hans G. Helms (Helms 1966) genannt.
} 


\section{Literatur}

Althusser, L. (2005). Pour Marx. Paris: Editions La Découverte.

Altvater, E. (2011). Das Ende des Kapitalismus, wie wir ihn kennen. Fernwald: Westphälisches Dampfboot. Badiou, A. (2011). Die kommunistische Hypothese. Leipzig: Merve.

Böhm-Bawerk, E. (1896). Zum Abschluß des Marxschen Systems, Staatswissenschaftliche Arbeiten. Festgaben für Karl Knies (S. 87-205). Berlin: Otto von Boenigk.

Bortkiewicz, L. (1906). Wertrechnung und Preisrechnung im Marxschen System. Archiv für Sozialwissenschaft, 23, 1-50.

Bourdieu, P. (1996). Reflexive Anthropologie. Frankfurt am Main: Suhrkamp.

Burns, T. (2017). Marx, the labour theory of value and the transformation problem. Capital \& Class, 41(3), 493-510.

Catephores, G. (1994). The imperious Austrian: Schumpeter as bourgeois marxist. New Left Review, I(205), 3-30.

Darwin, Ch (1872). The origin of species by means of natural selection, or the preservation of favoured races in the struggle for life (6. Aufl.). London: John Murray.

Descartes R., 1631 (1701), Inquisitio veritatis per lumen naturale.

Dosi, G. (1982). Technological paradigms and technological trajectories. A suggested interpretation of the determinants and directions of technical change. Research Policy, 11(3), 147-162.

Flaschel, P., \& Luchtenberg, S. (2012). Roads to social capitalism. Theory, evidence and policy. Cheltenham: Edward Elgar Publishing.

Flaschel, P., et al. (2018). Value, competition and exploitation. Cheltenham: Edward Elgar Publishing.

Foley, D. (1995). Where do we go from here. Discussion Papers (REL - Recherches Economiques de Louvain) 1995031. Louvain: Université catholique de Louvain, Institut de Recherches Economiques et Sociales (IRES).

Freeman, A., Kliman, A., \& Wells, J. (Hrsg.). (2004). The new value controversy and the foundations of economics. Cheltenham: Edward Elgar.

Fuchs, Ch (2016). Reading marx in the information age. New York: Routledge.

Gintis, H. (2016). Individuality and entanglement: the moral and material bases of social life. Princeton (New Jersey): Princeton University Press.

Goodwin, R. (1967). A growth cycle. In C.H. Feinstein (Hrsg.), Socialism, capitalism and economic growth. Cambridge: Cambridge University Press.

Goodwin, R. (1990). Schumpeter, Keynes and the theory of economic evolution, economia e banca. Annali Scientifici, 3, 69-94.

Goodwin, R., \& Punzo, L. (1987). The dynamics of a capitalist economy: a multisectoral approach. Oxford: Westview Press, Boulder, and Basil Blackwell,.

Gordon, D. (1978). Up and down the long roller coaster. In Union for Radical Political Economics (Hrsg.), U.S. Capitalism in crisis (S. 22-34). New York: URPE.

Gordon, D., Bowles, S., \& Weisskopf, Th (1985). Beyond the Wasteland: A Democratic Alternative to Economic Decline. London: Verso Books.

Gould, S. J. (2011). The Darwinian gentleman at marx's funeral: resolving evolution's oddest coupling. In S. J. Gould (Hrsg.), I have landed. The end of a beginning in natural history. Harvard: Belknap Press.

Gramsci, A. (1999). Prison notebooks. In Further selections from the prison notebooks. London: Electric Book Company.

Hanappi, H. (1986). The stages of industrial capitalism. In A. Vercelli, et al. (Hrsg.), Technological and social factors in long term fluctuations. Heidelberg, New York: Springer. University of Siena.

Hanappi, H. (1988). Die moderne Arbeitswerttheorie. Wiener Studien zur Politischen Ökonomie, Bd. 3. http://www.econ.tuwien.ac.at/hanappi/Wispo/wispo_3.pdf

Hanappi, H. (2006). Endogenous needs, values and technology. MPRA paper Nr. 28880. Munich: Munich Personal RePEc Archive.

Hanappi, H. (2013). The Neumann-Morgenstern Project. In H. Hanappi (Hrsg.), Game theory Relaunched. Rijeka: Intech publishers.

Hanappi, H. (2014). Bridges to babylon. From Keynesian macroeconomics to evolutionary macroeconomic simulation models. In L. Mamica \& P. Tridico (Hrsg.), Economic policy and the financial crisis. New York: Routledge.

Hanappi, H. (2015). Schumpeter and Goodwin. Journal of Evolutionary Economics, 25(1), 277-291.

Hanappi, H. (2017). Agent-based modelling. History-essence-future. PSL Quarterly Review, 70(283), $449-472$.

Hanappi, H. (2018). Capital after capitalism. World Review of Political Economy, 9(1), 61-79. 
Hanappi, H., \& Scholz-Wäckerle, M. (2017). Evolutionary political economy: content and method. Forum for Social Economics. https://doi.org/10.1080/07360932.2017.1287748.

Harrod, R. (1939). An essay in dynamic theory. The Economic Journal, 49(193), 14-33.

Helms, H. G. (1966). Die Ideologie der anonymen Gesellschaft. Max Stirners „Einziger“ und der Fortschritt des demokratischen Selbstbewußtseins vom Vormärz bis zur Bundesrepublik. Köln: DuMont Schauberg.

Hicks, J. (1937). Mr Keynes and the classics: a suggested interpretation. Econometrica, 5(2), 147-159.

Hilferding, R. (1910). Das Finanzkapital, Eine Studie zur jüngsten Entwicklung des Kapitalismus. Wien: Verlag der Wiener Volksbuchhandlung Ignaz Brand \& Co.

Jones, G. S. (2016). Karl Marx. Greatness and illusion. London: Allen Lane.

Kaldor, N. (1955). Alternative theories of distribution. Review of Economic Studies, 23, 83-100.

Kalecki, M. (1942). A theory of profits. Economic Journal, 52(206/207), 258-267.

Keynes, J. M. (1936). General theory of employment, interest and money. London: Palgrave Macmillan.

Kliman, A. (2007). Reclaiming Marx's capital: a refutation of the myth of inconsistency. Lanham: Lexington Books.

Leontieff, W. (1966). Input-output economics. Oxford: Oxford University Press.

Marglin, S. (1984). Growth, distribution, and prices. Cambridge: Harvard University Press.

Marx, K. (1852). Der 18. Brumaire des Louis Bonaparte. In Die Revolution. Eine Zeitschrift in zwanglosen Heften von Joseph Weydemeyer. 2. überarbeitete Auflage 1869 in Hamburg.

Marx, K. (1874). Kritik des Gothaer Programms. Die Neue Zeit, 1(18), 1890-1891.

Merlau-Ponty, M. (1955). Les aventures de la dialectique. Paris: Gallimard.

Morishima, M. (1973). Marx's economics. Cambridge: Cambridge University Press.

Neffe, J. (2017). Marx. Der Unvollendete. München: Bertelsmann.

Pasinetti, L. (1977). Lectures on the theory of production. New York: Columbia University Press.

Poulantzas, N. (2000). State, power, socialism. London: Verso.

Quesnay, F. (1758). Tableau économique, et maximes générales du governement économiques. Versailles.

Ramsey, F. (1928). A mathematical theory of saving. The Economic Journal, 38(152), 543-559.

Roemer, J. (1981). Analytical foundations of Marxian economic theory. Cambridge: Cambridge University Press.

Rosanda, R. (1974). Über die Dialektik von Kontinuität und Bruch. Zur Kritik revolutionärer Erfahrungen - Italien, Frankreich, Sowjetunion, Polen, China, Chile. Berlin: Suhrkamp. es 687.

Samuelson, P. (1947). Foundations of economic analysis. Harvard Economic Studies, Bd. 80.

Sargent, Th (1987). Macroeconomic Theory (2. Aufl.). Bingley: Emerald Group.

Schumpeter, J. (1911). Theorie der wirtschaftlichen Entwicklung. Berlin: Duncker \& Humblot.

Schumpeter, J. (1954). History of economic analysis. London: Allen Unwin.

Solow, R. (1956). A contribution to the theory of economic growth. Quarterly Journal of Economics, 70, 65-94.

Veneziani, R. (2010). Analytical Marxism. Journal of Economic Surveys. https://doi.org/10.1111/j.14676419.2010.00667.x.

Wagenknecht, S. (2016). Reichtum ohne Gier. Wie wir uns vor dem Kapitalismus retten. Frankfurt: Campus.

Wright, E. O. (2017). Reale Utopien. Wege aus dem Kapitalismus. Berlin: Suhrkamp.

Žižek, S. (2015). Ärger im Paradies. Vom Ende der Geschichte des Kapitalismus. Frankfurt am Main: Fischer Wissenschaft. 